\title{
Greater mastery is associated with lower depression risk in a large international cohort of people with multiple sclerosis over 2.5 years
}

\section{Sandra Neate}

The University of Melbourne School of Population Health: The University of Melbourne School of Population and Global Health

\section{Afaf Humam}

The University of Melbourne Department of Public Health: The University of Melbourne School of Population and Global Health

\section{Nupur Nag}

The University of Melbourne Department of Public Health: The University of Melbourne School of Population and Global Health

\section{George Jelinek}

The University of Melbourne Department of Public Health: The University of Melbourne School of Population and Global Health

\section{Steve Simpson-Yap ( $\sim$ steve.simpsonyap@unimelb.edu.au )}

The University of Melbourne School of Population Health: The University of Melbourne School of Population and Global Health https://orcid.org/0000-0001-6521-3056

\section{Research Article}

Keywords: mastery, multiple sclerosis, pwMS, depression risk

Posted Date: March 30th, 2021

DOI: https://doi.org/10.21203/rs.3.rs-319260/v1

License: (c) (i) This work is licensed under a Creative Commons Attribution 4.0 International License. Read Full License

Version of Record: A version of this preprint was published at Quality of Life Research on November 23rd, 2021. See the published version at https://doi.org/10.1007/s11136-021-03033-7. 


\section{Abstract}

Background: Mastery is the extent to which an individual perceives their life circumstances as being under their control and not predominantly influenced by external factors. The relationship of mastery with clinical outcomes in people with multiple sclerosis (pwMS) has not been well-researched. We assessed the relationships of mastery with fatigue, disability, relapse number, and depression risk among pwMS over 2.5 years' follow-up.

Methods: Data from the Health Outcomes and Lifestyle in a Sample of people with Multiple sclerosis (HOLISM) study, among 839 participants who completed the 2.5 and 5-year reviews, were analysed. Mastery was measured by the Pearlin Mastery Scale, fatigue by Fatigue Severity Scale, depression risk by Patient Health Questionnaire-9, and disability by PatientDetermined Disease Steps, and diagnosed relapse number in the previous 12 months was queried. Cross-sectional and prospective analyses were undertaken by log-binomial, log-multinomial, and Poisson regression, as appropriate, adjusted for relevant confounders.

Results: Cross-sectionally, pwMS with the highest quartile mastery (>25/28) had $90 \%$ lower depression risk, $60 \%$ lower frequency of clinically significant fatigue, and $77 \%$ fewer with severe disability, all largely robust to adjustment. Prospectively, those in the top two quartiles of mastery $(>21-25,>25)$ had $66 \%$ and $74 \%$ lower depression risk, robust to adjustment. No significant associations were seen prospectively for change in fatigue, disability, or relapse number, however, and no robust associations of mastery with relapse number.

Conclusions: Prospectively, a protective relationship of mastery with subsequent risk of depression was observed, suggesting this may be a point of intervention to improve wellbeing in pwMS.

\section{Introduction}

Multiple sclerosis (MS) is an autoimmune neurodegenerative disease that affects the central nervous system. The multiple physical, emotional and social challenges faced by people with MS (pwMS) have a significant impact on feelings of control over their lives. The sense of autonomy and agency, that is, the capacity to act independently and make one's own choices, may be impaired by the impact of these challenges. The concepts of autonomy and agency fit into the conceptual framework referred to as mastery, defined broadly as the sense of feeling in control of one's life [1]. Mastery also encompasses how a person views their life, whether they feel they can influence their own life and that their outcomes are not fatalistically controlled. Mastery fits within a similar framework as concepts such as "self-efficacy" or "coping style". Self-efficacy is the confidence in one's own capabilities to manage situations and overcome challenges [2]. These related concepts all involve how an individual perceives themselves, their response to adversity, and how they cope.

Studies examining self-efficacy and self-esteem in pwMS have identified that improved self-esteem is associated with the use of strategies for improved coping and that feelings of self-worth are linked to the ability to handle challenging life situations [3]. Self-efficacy in MS has been identified as a determinant of several outcomes including perceived health status $[4 ; 5]$, ability to adjust to illness [6], and health-related quality of life [7]. Mastery has also been associated with a dosedependent improvement in both mental and physical health-related quality of life in pwMS[8].

With respect to specific health outcomes in MS, an improved sense of mastery has been demonstrated to ameliorate fatigue, the distress associated with fatigue, and the subsequent inability to perform tasks [9]. The treatment of depression in MS has been shown to improve the individual's sense of mastery, sense of purpose in life, and their level of self-acceptance [10]. However, the impact of mastery on other clinical outcomes in PwMS has not been well-explored. This study therefore assessed the cross-sectional and prospective relationships of mastery with fatigue, level of disability, relapse number, and depression risk over 2.5 years in an international cohort of pwMS.

\section{Methods}




\section{Participants}

Data from the Health Outcomes and Lifestyle In a Sample of people with Multiple sclerosis (HOLISM) study were analysed. The methodology of the HOLISM study has been described previously [11; 12]. Briefly, participants were recruited through Web 2.0 platforms and invited to complete an online survey. Consenting individuals at least 18 years of age proceeded to the survey and were subsequently invited to complete follow-up surveys at 2.5-year intervals thereafter. All analyses are constrained to persons reporting a physician diagnosis of MS at baseline. Here, we report analyses of data from the 2.5-year time point (referred to as the 2.5-year review) when mastery was first queried and follow-up 2.5 years later, that is 5 years from the baseline survey (referred to as the 5-year review).

Ethics approval was given by The University of Melbourne's Health Sciences and Human Ethics Sub-Committee (HESC 1545102).

\section{Data collection and tools}

Participants completed a multi-item questionnaire capturing demographic, lifestyle, and clinical characteristics. Data included age, sex, marital status, country of residence, level of education, perceived relative socioeconomic status (SES) [13], height and weight from which body mass index (BMI) was calculated, physical activity (International Physical Activity Questionnaire, IPAQ [14]), number of people in their core social support network, MS type, baseline number of treated comorbidities[15], and medication use, as described previously [12].

The primary exposure of interest was mastery, measured by the Pearlin Mastery Scale (PMS) [1]. The scale consists of seven statements, each measured on a 4-point Likert scale, where 1 equals strongly agree and 4 strongly disagree. Responses were summated to realise a total PMS score ranging from 7 (low mastery) to 28 (high mastery), and then categorised into quartiles.

Four clinical outcomes were assessed at each timepoint by validated tools. Fatigue was assessed by the Fatigue Severity Scale (FSS) [16], where clinically significant fatigue was defined as mean FSS score $>5$. Disability was measured using the Patient-Determined Disease Steps (PDDS) [17], and categorised as mild (PDSS=0-3), moderate (PDSS $>3-6$ ), and severe (PDSS >6). Depression risk was assessed by the Patient Health Questionnaire-9 (PHQ-9) [18], a score>9 indicating depression risk. Doctor-diagnosed relapse number in the previous 12 months was also queried. Participants were also asked whether they were experiencing ongoing symptoms due to recent relapse in the preceding 30 days.

\section{Data analysis}

Characteristics of mastery at 2.5-year review were assessed by linear regression, adjusted for ongoing symptoms from recent relapse, and further adjusted for age, sex, education, support number, PDDS, clinically significant fatigue, depression risk, and treated comorbidity number. For cross-sectional analyses at 2.5-year review, clinically significant fatigue, disability, and depression risk were assessed by log-binomial regression, and relapse number by Poisson regression. All models except for relapse number were adjusted at minimum for whether participants were experiencing ongoing symptoms from recent relapse. Full multivariable models were constructed and covariates that materially affected models developed, these including age, sex, MS type, PDDS, clinically significant fatigue, depression risk, and antidepressant medication use, as appropriate for the outcome.

For change analyses from the 2.5 to 5 -year reviews, dichotomous outcomes (fatigue, depression risk) were evaluated by logbinomial regression, as the proportion of pwMS without the outcome at either timepoint compared with the subset who developed the outcome at 5-year review (gain of outcome), and the proportion of pwMS with the outcome at both timepoints compared with the subset who did not have that outcome at 5-year review (loss of outcome), a method we have used 
previously [19]. Change in disability and relapse number were evaluated as a difference in the outcome, with "increasing", "decreasing" or "the same" evaluated as polychotomous outcomes by log-multinomial regression. For disability, fatigue, and depression analyses, all models were adjusted for the 2.5-year values of the outcome variable, as well as whether participants were experiencing ongoing symptoms from recent relapse at baseline and follow-up.

All analyses were conducted using Stata SE/15.0 (StataCorp, College Station, TX, USA).

\section{Results}

Of 2,466 baseline participants, 1,401 (58.4\%) completed the survey at 2.5-year review. Of the 1,401 participants, 839 (59.9\%) also completed the 5-year review and are included in this analyses.

Of the 839 participants, $n=793(94.5 \%)$ completed the Pearlin Mastery Scale (PMS) questions. Respondents to the PMS were mostly female (82.4\%), had a mean age of 51 years, and $64.2 \%$ had between two and five support people in their lives. The largest proportion of participants lived in Australia/New Zealand (47.3\%), most had completed university (69.8\%), and were of higher perceived socioeconomic status (54.0\%). Respondents were predominantly diagnosed with benign or relapsingremitting MS (RRMS; 70.7\%), were of normal BMI (64.1\%) and reported no treated comorbidities (62.4\%). The average disease duration from onset at 5 -year review was 19.0 years $(S D=10.1)$.

Mastery was significantly higher among those with postgraduate degrees, higher SES, more than six members in their social support network, and those who were physically active, while mastery was lower among those of progressive MS type, severe disability, with clinically significant fatigue, and with depression risk, all robust to adjustment for age, sex, education, support number, PDDS, clinically significant fatigue, depression risk, and comorbidity number. Other characteristics of mastery are shown in Table 1.

Table 1. Characteristics of mastery at 2.5-year review. 


\begin{tabular}{|c|c|c|c|}
\hline Characteristics & $\mathrm{n} / \mathrm{N}(\%)$ & $a \beta(95 \% C l)^{a}$ & $a \beta(95 \% \mathrm{Cl})^{\mathrm{b}}$ \\
\hline Sex & 140/793 (17.7\%) & 0.00 [Reference] & 0.00 [Reference] \\
\hline Male & $653 / 793(82.4 \%)$ & $-0.19(-0.95,0.57)$ & $0.21(-0.49,0.92)$ \\
\hline Female & & $p=0.62$ & $p=0.55$ \\
\hline Age & $202 / 793$ (25.5\%) & 0.00 [Reference] & 0.00 [Reference] \\
\hline $18.0-42.9$ & 198/793 (25.0\%) & $0.11(-0.71,0.93)$ & $0.41(-0.34,1.16)$ \\
\hline$>42.9-51.0$ & $201 / 793$ (25.4\%) & $-0.11(-0.92,0.70)$ & $0.62(-0.15,1.39)$ \\
\hline$>51.0-58.5$ & $192 / 793(24.2 \%)$ & $-0.55(-1.38,0.28)$ & $0.51(-0.30,1.31)$ \\
\hline$>58.5$ & & $p=0.17$ & $p=0.14$ \\
\hline \multicolumn{4}{|l|}{ Trend: } \\
\hline Education level & $122(15.4 \%)$ & 0.00 [Reference] & 0.00 [Reference] \\
\hline Up to secondary & $117(14.8 \%)$ & $1.29(0.24,2.33)$ & $1.15(0.19,2.11)$ \\
\hline Vocational training & $305(38.5 \%)$ & $1.57(0.70,2.43)$ & $1.06(0.24,1.88)$ \\
\hline Bachelor's degree & $248(31.3 \%)$ & $2.00(1.10,2.89)$ & $1.46(0.62,2.30)$ \\
\hline Post-graduate degree & & $p<0.001$ & $p=0.003$ \\
\hline Trend: & & & \\
\hline
\end{tabular}

Perceived relative socioeconomic status

Lower
Same
Higher
Trend:
Number of people in core social support network

$\begin{array}{llll}0 & 19 / 770(2.5 \%) & 0.00[\text { Reference] } & 0.00 \text { [Reference] } \\ 1 & 157 / 770(20.4 \%) & 0.63(-1.35,2.60) & 0.89(-0.84,2.61) \\ 2-5 & 494 / 770(64.2 \%) & 1.52(-0.38,3.42) & 1.21(-0.45,2.87) \\ 6-9 & 61 / 770(7.9 \%) & 2.88(0.75,5.02) & 2.25(0.37,4.13) \\ 10+ & 39 / 770(5.1 \%) & 3.07(0.80,5.34) & 2.65(0.66,4.65) \\ \text { Trend: } & & \mathbf{p}<0.001 & p<0.001 \\ \text { BMI } & 508 / 793(64.1 \%) & 0.00[\text { Reference] } & 0.00[\text { Reference] } \\ \text { Normal }(>18.5-25) & 164 / 793(20.7 \%) & -1.18(-1.91,-0.46) & -0.37(-1.05,0.30) \\ \text { Overweight }(>25-30) & 121 / 793(15.3 \%) & -1.16(-1.98,-0.34) & 0.38(-0.44,1.20) \\ \text { Obese }(>30) & & p<0.001 & p=0.70 \\ \text { Trend: } & & & 0.00[\text { Reference] } \\ \text { IPAQ } & 222 / 778(28.5 \%) & 0.00[\text { Reference] } & 0.82(0.15,1.50) \\ \text { Inactive } & 352 / 778(45.1 \%) & \mathbf{2 . 0 3 ( 1 . 3 6 , 2 . 7 1 )} & .\end{array}$

Page 5/14 
Minimally active

Active

Trend:

MS type

Benign/RRMS

SPMS/PPMS/PRMS

Unsure/Other

Duration since MS onset, years

$\begin{array}{ll}2.88-8.01 & 198(25.0 \%) \\ >8.01-13.92 & 204(25.8 \%) \\ >13.92-22.22 & 197(24.9 \%) \\ >22.22-53.92 & 193(24.4 \%) \\ \text { Trend: } & \\ \text { PDDS } & 486(61.4 \%) \\ \text { Normal/mild } & 226(28.5 \%) \\ \text { Moderate } & 80(10.1 \%)\end{array}$

Severe

Trend:

Clinically significant fatigue

No

Yes

$319 / 759(42.0 \%)$

440/759 (58.0\%)

0.00 [Reference]

0.00 [Reference]

$-3.39(-3.94,-2.83)$

$-2.08(-2.69,-1.48)$

$p<0.001$

MS immunomodulatory medication use

None

Interferon- $\beta$

Other $^{\mathrm{C}}$

Depression risk (PHQ-9)

No

Yes

$622 / 762$ (81.6\%)

140/762 (18.4\%)

77/793 (9.7\%)

0.00 [Reference]

0.00 [Reference]

$272 / 793$ (34.3\%)

$-0.46(-1.47,0.54)$

$-0.64(-1.58,0.29)$

$-0.51(-1.14,0.12)$

$-0.46(-1.06,0.13)$

$p<0.001$

Number of treated comorbidities

0
1
2
3 or more

0.00 [Reference]

$-2.92(-3.64,-2.19)$

$p<0.001$

$\begin{array}{lll}495 / 793(62.4 \%) & 0.00 \text { [Reference] } & 0.00 \text { [Reference] } \\ 174 / 793(21.9 \%) & -1.23(-1.93,-0.52) & -0.51(-1.17,0.16) \\ 88 / 793(11.1 \%) & -1.86(-2.79,-0.94) & -0.68(-1.58,0.22) \\ 36 / 793(4.5 \%) & -2.84(-4.22,-1.46) & -1.31(-2.64,0.02) \\ & p<0.001 & p=0.013\end{array}$


Trend:

Prescription antidepressant medication use

$\begin{array}{llll}\text { No } & 671(84.6 \%) & 0.00 \text { [Reference] } & 0.00 \text { [Reference] } \\ \text { Yes } & 122(15.4 \%) & -\mathbf{- 2 . 4 0 ( - 3 . 1 9 , - 1 . 6 2 )} & -0.12(-0.97,0.73) \\ & & p<0.001 & p=0.78\end{array}$

All models by linear regression, estimating $\beta(95 \% \mathrm{Cl})$. Results in boldface denote statistical significance $(p<0.05)$.

a Multivariable model adjusted for ongoing symptoms of recent relapse.

b Multivariable model adjusted for ongoing symptoms of recent relapse and further adjusted for age, sex, education, support number, PDDS, clinically significant fatigue, depression risk, and treated comorbidity number.

c Other DMTs include glatiramer acetate, alemtuzumab, cladribine, daclizumab, dimethyl fumarate, fingolimod, laquinimod, rituximab, teriflunomide, and natalizumab.

Abbreviations: BMI = Body mass index; IPAQ = International Physical Activity Questionnaire; PHQ-9 = Patient Health Questionnaire 9; PDDS = Patient Determined Disease Steps, PPMS = Primary progressive MS; PRMS = Progressiverelapsing MS; RRMS = Relapsing-remitting MS; SPMS = Secondary-progressive MS .

\section{Cross-sectional analyses of outcomes at 2.5-year review}

\section{Depression risk}

A dose-dependent inverse association between mastery and depression risk was found, persisting upon adjustment for age, sex, clinically significant fatigue, disability, number of treated comorbidities, and prescription antidepressant medication use (Supplemental Table 1). Participants with the highest quartile higher mastery score had $90 \%$ lower frequency of depression risk compared to those the lowest mastery score.

\section{Fatigue}

The majority (82.8\%) of participants with a mastery score of 7-19 had clinically significant fatigue (Supplemental Table 1). FSS and mastery were inversely associated, persisting on adjustment for ongoing symptoms of recent relapse, age, sex, disability, depression risk, and number of treated comorbidities, and showing a dose-dependent association. Participants scoring in the top two quartiles of mastery showed $31 \%$ and $50 \%$, respectively, lower frequencies of clinically significant fatigue.

\section{Relapse number}

In univariable analyses, those with higher mastery score had significantly fewer relapses in the preceding 12 months (Supplemental Table 1). On adjustment for age, sex, clinically significant fatigue, disability, depression risk, and number of treated comorbidities, however, this association was attenuated and lost dose-dependency.

\section{Disability}

\section{An inverse association was found between mastery and both moderate to mild disability and mild to severe disability (Supplemental Table 2). On adjustment for age, sex, clinically significant fatigue, depression}




\section{risk, and number of treated comorbidities, however, only the association of mastery with severe disability persisted.}

\section{Prospective change analyses}

\section{Change in depression risk}

Mastery above the median was associated with significantly lower risk of developing depression risk, this attenuating only slightly on adjustment such that those in the top two quartiles had $63 \%$ and $68 \%$ lower risk of developing depression risk, respectively (Table 2). Consolidating the top and bottom two quartiles found that those with mastery above the median had $70 \%$ lower risk of developing depression risk, persisting on adjustment. No association with losing depression risk was seen, however.

Table 2. Mastery \& change in depression risk

\begin{tabular}{|c|c|c|c|c|c|c|c|c|}
\hline \multirow{2}{*}{$\begin{array}{l}\text { n (row \%) } \\
\text { Always } \\
\text { depression } \\
\text { risk }\end{array}$} & \multirow[b]{2}{*}{$\begin{array}{l}\text { Stops } \\
\text { depression } \\
\text { risk }\end{array}$} & \multicolumn{2}{|c|}{$\begin{array}{l}\text { Loss of depression } \\
\text { risk }\end{array}$} & \multicolumn{2}{|l|}{ n (row \%) } & \multicolumn{3}{|c|}{ Gain of depression risk } \\
\hline & & $\begin{array}{l}\text { aRR } \\
(95 \% \\
\mathrm{Cl})^{\mathrm{a}}\end{array}$ & $\begin{array}{l}\text { aRR }(95 \% \\
\mathrm{CI})^{\mathrm{b}}\end{array}$ & $\begin{array}{l}\text { Never } \\
\text { depression } \\
\text { risk }\end{array}$ & $\begin{array}{l}\text { Develops } \\
\text { depression } \\
\text { risk }\end{array}$ & $\begin{array}{l}\text { aRR } \\
(95 \% \\
\mathrm{Cl})^{\mathrm{a}}\end{array}$ & $\begin{array}{l}\text { aRR }(95 \% \\
\mathrm{Cl})^{\mathrm{b}}\end{array}$ & \multirow[b]{2}{*}{$\begin{array}{l}1.00 \\
\text { [Reference] }\end{array}$} \\
\hline & $53(58.9 \%)$ & $\begin{array}{l}37 \\
(41.1 \%)\end{array}$ & $\begin{array}{l}1.00 \\
\text { [Reference] }\end{array}$ & $\begin{array}{l}1.00 \\
\text { [Reference] }\end{array}$ & $\begin{array}{l}107 \\
(83.0 \%)\end{array}$ & $\begin{array}{l}22 \\
(17.1 \%)\end{array}$ & $\begin{array}{l}1.00 \\
\text { [Reference] }\end{array}$ & \\
\hline $\begin{array}{l}7-19 \\
>19-21\end{array}$ & $\begin{array}{l}14(56.0 \%) \\
10(66.7 \%)\end{array}$ & $\begin{array}{l}11 \\
(44.0 \%)\end{array}$ & $\begin{array}{l}1.05(0.63 \\
1.75)\end{array}$ & $\begin{array}{l}1.04(0.66 \\
1.66)\end{array}$ & $\begin{array}{l}100 \\
(83.3 \%)\end{array}$ & $\begin{array}{l}20 \\
(16.7 \%)\end{array}$ & $\begin{array}{l}0.98(0.56 \\
1.71)\end{array}$ & $\begin{array}{l}0.99(0.55 \\
1.77)\end{array}$ \\
\hline $\begin{array}{l}>21-25 \\
>25-28\end{array}$ & $4(57.1 \%)$ & $\begin{array}{l}5 \\
(33.3 \%)\end{array}$ & $\begin{array}{l}0.80(0.38 \\
1.67)\end{array}$ & $\begin{array}{l}0.90(0.43 \\
1.87)\end{array}$ & $\begin{array}{l}181 \\
(94.3 \%)\end{array}$ & $\begin{array}{l}11 \\
(5.7 \%)\end{array}$ & $\begin{array}{l}0.34(0.17 \\
0.67)\end{array}$ & $\begin{array}{l}0.37(0.18, \\
0.75)\end{array}$ \\
\hline Trend: & & $\begin{array}{l}3 \\
(42.9 \%)\end{array}$ & $\begin{array}{l}0.99(0.43 \\
2.29) \\
p=0.75\end{array}$ & $\begin{array}{l}1.20(0.43 \\
3.38) \\
p=0.85\end{array}$ & $\begin{array}{l}156 \\
(95.7 \%)\end{array}$ & $\begin{array}{l}7 \\
(4.3 \%)\end{array}$ & $\begin{array}{l}0.26(0.11, \\
0.58) \\
p<0.001\end{array}$ & $\begin{array}{l}0.32(0.14 \\
0.75) \\
p=0.001\end{array}$ \\
\hline $\begin{array}{l}\text { Mastery } \\
7-21 \\
>21-28\end{array}$ & $\begin{array}{l}67(58.3 \%) \\
14(63.6 \%)\end{array}$ & $\begin{array}{l}48 \\
(41.7 \%) \\
8 \\
(36.4 \%)\end{array}$ & $\begin{array}{l}1.00 \\
\text { [Reference] } \\
0.85(0.48 \\
1.52) \\
p=0.58\end{array}$ & $\begin{array}{l}1.00 \\
\text { [Reference] } \\
0.98(0.53 \\
1.80) \\
p=0.95\end{array}$ & $\begin{array}{l}207 \\
(83.1 \%) \\
337 \\
(94.9 \%)\end{array}$ & $\begin{array}{l}42 \\
(16.9 \%) \\
18 \\
(5.1 \%)\end{array}$ & $\begin{array}{l}\begin{array}{l}1.00 \\
\text { [Reference] }\end{array} \\
0.30(0.18 \\
0.51) \\
p<0.001\end{array}$ & $\begin{array}{l}1.00 \\
\text { [Reference] } \\
0.35(0.21, \\
0.60) \\
p<0.001\end{array}$ \\
\hline $\begin{array}{l}\text { a Multivaria } \\
\text { of relapse a } \\
\text { b Multivaria } \\
\text { number of } t\end{array}$ & $\begin{array}{l}\text { log-multin } \\
\text { log binomi } \\
\text { ongoing sy } \\
\text { log binomi } \\
\text { ted comorb }\end{array}$ & $\begin{array}{l}\text { gressi } \\
\text { oms of } \\
\text { esressi }\end{array}$ & $\begin{array}{l}\text { hodel adju } \\
\text { apse. } \\
\text { odel adju } \\
\text { e clinically }\end{array}$ & $\begin{array}{l}\text { for baseli } \\
\text { for baseli } \\
\text { iificant fa }\end{array}$ & $\begin{array}{l}\text { epression } \\
\text { epression } \\
\text { and pres }\end{array}$ & $\begin{array}{l}\text { PHQ-9) } \\
\text { PHQ-9) } \\
\text { on antic }\end{array}$ & $\begin{array}{l}\text { seline ong } \\
\text { e, sex, bas } \\
\text { ressant me }\end{array}$ & $\begin{array}{l}\text { symptoms } \\
\text { e disability, } \\
\text { ation use. }\end{array}$ \\
\hline
\end{tabular}

\section{Change in clinically significant fatigue, relapse number, and disability}

Mastery had no significant association with change in clinically significant fatigue from 2.5 year to 5 -year review (Table 3). A positive trend was seen for resolution of fatigue, driven by those in the top quartile of mastery, but this attenuated and became nonsignificant on adjustment. Similarly, a reciprocal inverse trend was seen for developing fatigue but this disappeared on adjustment. 
In univariable models, those with 2.5-year mastery in the top quartile had 59\% lower risk of increasing their diagnosed relapse number, though this association attenuated on adjustment. No association was seen for decrease in relapse number, however.

No associations were seen between 2.5-year baseline mastery and subsequent increase or decrease in disability.

Table 3. Mastery \& change in clinically significant fatigue, relapse number, and disability. 


\begin{tabular}{|c|c|c|c|c|c|c|c|c|}
\hline \multicolumn{4}{|c|}{$\begin{array}{l}\text { Change in clinically significant } \\
\text { fatigue }\end{array}$} & \multirow{2}{*}{\multicolumn{2}{|c|}{ n (row \%) }} & \multirow{2}{*}{\multicolumn{2}{|c|}{ Gain of fatigue }} & \\
\hline \multicolumn{2}{|l|}{ n (row \%) } & \multicolumn{2}{|c|}{ Loss of fatigue } & & & & & \\
\hline $\begin{array}{l}\text { Always } \\
\text { fatigued }\end{array}$ & $\begin{array}{l}\text { Stops } \\
\text { fatigue }\end{array}$ & $\begin{array}{l}\text { aRR } \\
(95 \% \\
\mathrm{Cl})^{a}\end{array}$ & $\begin{array}{l}\text { aRR }(95 \% \\
\mathrm{CI})^{\mathrm{b}}\end{array}$ & $\begin{array}{l}\text { Never } \\
\text { fatigued }\end{array}$ & $\begin{array}{l}\text { Starts } \\
\text { fatigue }\end{array}$ & $\begin{array}{l}\text { aRR }(95 \% \\
\mathrm{Cl})^{\mathrm{a}}\end{array}$ & $\begin{array}{l}\text { aRR }(95 \% \\
\mathrm{Cl})^{\mathrm{b}}\end{array}$ & \\
\hline & $\begin{array}{l}159 \\
(87.4 \%)\end{array}$ & $\begin{array}{l}23 \\
(12.6 \%)\end{array}$ & $\begin{array}{l}1.00 \\
\text { [Reference] }\end{array}$ & $\begin{array}{l}1.00 \\
\text { [Reference] }\end{array}$ & & & $\begin{array}{l}1.00 \\
\text { [Reference] }\end{array}$ & $\begin{array}{l}1.00 \\
\text { [Reference] }\end{array}$ \\
\hline $\begin{array}{l}7-19 \\
>19-21\end{array}$ & $\begin{array}{l}73 \\
(79.4 \%)\end{array}$ & $\begin{array}{l}19 \\
(20.7 \%)\end{array}$ & $\begin{array}{l}1.64(0.95 \\
2.84)\end{array}$ & $\begin{array}{l}1.61(0.92 \\
2.84)\end{array}$ & $\begin{array}{l}40(78.4 \%) \\
93(86.9 \%)\end{array}$ & $\begin{array}{l}11(21.6 \%) \\
14(13.1 \%)\end{array}$ & $\begin{array}{l}0.72(0.36 \\
1.48)\end{array}$ & $\begin{array}{l}0.82(0.38 \\
1.74)\end{array}$ \\
\hline$>21-25$ & $\begin{array}{l}85 \\
(86.7 \%)\end{array}$ & $\begin{array}{l}13 \\
(13.3 \%)\end{array}$ & $\begin{array}{l}1.05(0.56 \\
1.98)\end{array}$ & $\begin{array}{l}0.83(0.43 \\
1.61)\end{array}$ & $94(81.7 \%)$ & $21(18.3 \%)$ & $\begin{array}{l}0.43(0.22 \\
0.85)\end{array}$ & $\begin{array}{l}0.55(0.29 \\
1.05)\end{array}$ \\
\hline Trend: & $\begin{array}{l}40 \\
(72.7 \%)\end{array}$ & $\begin{array}{l}15 \\
(27.3 \%)\end{array}$ & $\begin{array}{l}1.96(1.11 \\
3.47)\end{array}$ & $\begin{array}{l}1.54(0.86 \\
2.76)\end{array}$ & & & $\begin{array}{l}0.63(0.34 \\
1.15)\end{array}$ & $\begin{array}{l}0.76(0.39 \\
1.47)\end{array}$ \\
\hline & & & $p=0.088$ & $p=0.63$ & & & $p=0.15$ & $p=0.56$ \\
\hline
\end{tabular}

\section{Change in relapse number}

\begin{tabular}{|c|c|c|c|c|c|c|c|}
\hline \multicolumn{3}{|l|}{$\mathrm{n}($ row \%) } & \multicolumn{2}{|c|}{ Relapse decrease } & \multicolumn{2}{|c|}{ Increase relapse } & \\
\hline $\begin{array}{l}\text { Relapse } \\
\text { decrease }\end{array}$ & $\begin{array}{l}\text { Stable } \\
\text { relapse }\end{array}$ & $\begin{array}{l}\text { Increase } \\
\text { relapse }\end{array}$ & $\begin{array}{l}\mathrm{RR}(95 \% \\
\mathrm{Cl})\end{array}$ & $\begin{array}{l}\text { aRR }(95 \% \\
\mathrm{Cl})^{\mathrm{c}}\end{array}$ & $\begin{array}{l}\mathrm{RR}(95 \% \\
\mathrm{Cl})\end{array}$ & $\begin{array}{l}\text { aRR }(95 \% \\
\mathrm{Cl})^{\mathrm{c}}\end{array}$ & \\
\hline & $\begin{array}{l}34 \\
(15.3 \%)\end{array}$ & $\begin{array}{l}163 \\
(73.4 \%)\end{array}$ & $25(11.3 \%)$ & $\begin{array}{l}1.00 \\
\text { [Reference] }\end{array}$ & $\begin{array}{l}1.00 \\
\text { [Reference] }\end{array}$ & $\begin{array}{l}1.00 \\
\text { [Reference] }\end{array}$ & $\begin{array}{l}1.00 \\
\text { [Reference] }\end{array}$ \\
\hline $\begin{array}{l}7-19 \\
>19-21\end{array}$ & $\begin{array}{l}13 \\
(9.1 \%)\end{array}$ & $\begin{array}{l}112 \\
(78.3 \%)\end{array}$ & $22(10.4 \%)$ & $\begin{array}{l}0.79(0.43 \\
1.46)\end{array}$ & $\begin{array}{l}0.91(0.40 \\
2.06)\end{array}$ & $\begin{array}{l}1.07(0.61 \\
1.88)\end{array}$ & $\begin{array}{l}1.00(0.57 \\
1.74)\end{array}$ \\
\hline & $\begin{array}{l}24 \\
(11.4 \%)\end{array}$ & $\begin{array}{l}165 \\
(78.2 \%)\end{array}$ & $8(4.6 \%)$ & $\begin{array}{l}0.96(0.58 \\
1.59)\end{array}$ & $\begin{array}{l}1.31(0.69 \\
2.49)\end{array}$ & $\begin{array}{l}0.93(0.54 \\
1.59)\end{array}$ & $\begin{array}{l}0.91(0.52 \\
1.60)\end{array}$ \\
\hline Trend: & $\begin{array}{l}17 \\
(9.8 \%)\end{array}$ & $\begin{array}{l}148 \\
(85.6 \%)\end{array}$ & & $\begin{array}{l}0.79(0.45 \\
1.40)\end{array}$ & $\begin{array}{l}1.19(0.57 \\
2.50)\end{array}$ & $\begin{array}{l}0.41(0.19, \\
0.89)\end{array}$ & $\begin{array}{l}0.53(0.25 \\
1.12)\end{array}$ \\
\hline & & & & $p=0.55$ & $p=0.57$ & $p=0.026$ & $p=0.25$ \\
\hline
\end{tabular}

\section{Change in disability}

\begin{tabular}{|c|c|c|c|c|c|c|c|}
\hline \multicolumn{3}{|l|}{ n (row \%) } & \multicolumn{2}{|c|}{ Disability decrease } & \multicolumn{2}{|c|}{ Increase disability } & \\
\hline $\begin{array}{l}\text { Disability } \\
\text { decrease }\end{array}$ & $\begin{array}{l}\text { Stable } \\
\text { disability }\end{array}$ & $\begin{array}{l}\text { Increase } \\
\text { disability }\end{array}$ & $\begin{array}{l}\text { aRR }(95 \% \\
\mathrm{Cl})^{\mathrm{a}}\end{array}$ & $\begin{array}{l}\text { aRR }(95 \% \\
\mathrm{Cl}^{\mathrm{d}}\end{array}$ & $\begin{array}{l}\text { aRR }(95 \% \\
\mathrm{Cl})^{\mathrm{a}}\end{array}$ & $\begin{array}{l}\text { aRR }(95 \% \\
\text { Cl) }\end{array}$ & \\
\hline Mastery & $\begin{array}{l}12 \\
(5.1 \%)\end{array}$ & $\begin{array}{l}196 \\
(83.1 \%)\end{array}$ & $28(11.9 \%)$ & $\begin{array}{l}1.00 \\
\text { [Reference] }\end{array}$ & $\begin{array}{l}1.00 \\
\text { [Reference] }\end{array}$ & $\begin{array}{l}1.00 \\
\text { [Reference] }\end{array}$ & $\begin{array}{l}1.00 \\
\text { [Reference] }\end{array}$ \\
\hline $\begin{array}{l}\text { 7-19 } \\
>19-21\end{array}$ & 8 (5.1\%) & $\begin{array}{l}132 \\
(84.1 \%)\end{array}$ & $15(6.8 \%)$ & $\begin{array}{l}0.96(0.41, \\
2.26)\end{array}$ & $\begin{array}{l}0.77(0.35 \\
1.66)\end{array}$ & $\begin{array}{l}0.90(0.52, \\
1.58)\end{array}$ & $\begin{array}{l}0.79(0.45 \\
1.38)\end{array}$ \\
\hline$>21-25$ & $\begin{array}{l}10 \\
(4.6 \%)\end{array}$ & $\begin{array}{l}195 \\
(88.6 \%)\end{array}$ & $13(7.3 \%)$ & $\begin{array}{l}0.85(0.38 \\
1.90)\end{array}$ & $\begin{array}{l}0.57(0.24 \\
1.35)\end{array}$ & $\begin{array}{l}0.59(0.33 \\
1.06)\end{array}$ & $\begin{array}{l}0.56(0.30 \\
1.06)\end{array}$ \\
\hline $\begin{array}{l}>25-28 \\
\text { Trend: }\end{array}$ & $6(3.4 \%)$ & $\begin{array}{l}160 \\
(89.4 \%)\end{array}$ & & $\begin{array}{l}0.61(0.24 \\
1.58)\end{array}$ & $\begin{array}{l}0.51(0.20 \\
1.31)\end{array}$ & $\begin{array}{l}0.69 \text { (0.36, } \\
1.31)^{(.6))}\end{array}$ & $\begin{array}{l}0.68 \text { (0.34, } \\
1.37)\end{array}$ \\
\hline & & & & $p=0.30$ & $p=0.80$ & $p=0.11$ & $p=0.32$ \\
\hline
\end{tabular}

All models by log-binomial regression, estimating risk ratio (RR) and adjusted risk ratios (aRR) (95\% Cl). Results in boldface denote statistical significance.

a Multivariable log-binomial regression model adjusted for ongoing symptoms of recent relapse at each timepoint. 
b Multivariable log-binomial regression model adjusted for ongoing symptoms of recent relapse at each timepoint, and baseline age, sex, disability, treated comorbidity number, and depression risk.

d Adjusted for baseline age, sex, disability, fatigue, treated comorbidity number, and depression risk.

d Multivariable log-binomial regression model adjusted for ongoing symptoms of recent relapse at each timepoint, and baseline age, sex, fatigue, treated comorbidity number, and depression risk.

\section{Discussion}

We have shown that higher mastery is cross-sectionally associated with lower frequencies of depression risk, clinically significant fatigue, and severe disability, robust to adjustment, and prospectively with a lower risk of developing depression risk over 2.5 years' follow-up. No prospective associations were seen for loss of depression risk, nor with any change in fatigue or disability, and no robust associations were seen for relapse number, either cross-sectionally or prospectively. These results suggest that improving mastery could be a point of intervention to prevent pwMS from developing depression, but associations with other clinical outcomes may reflect reverse causality.

Depression is a prevalent and debilitating symptom of MS with approximately $50 \%$ or more of pwMS experiencing a depressive disorder [20]. Our most important finding was that higher levels of mastery were associated with up to $70 \%$ reduced frequency of depression risk cross-sectionally and 60-70\% lower risk of developing depression risk prospectively. These results are in accord with our earlier studies showing greater mastery was associated with significantly better mental health-related quality of life [8]. It is also consistent with other studies that demonstrated inverse associations in self-efficacy scores with depressions scores in pwMS [21; 22].

Depression in MS is distinct from that seen in the general population, being considered potentially a comorbidity distinct from MS but occurring more frequently than in the general population, a symptom of MS, a common outcome with MS resulting from the same inflammatory aetiologic factors, and/or a consequence of the effects of MS and its clinical progression[23]. Regardless of its genesis, there is a potent role for stress in realising depression in pwMS, since peripheral inflammation can disrupt normal mood via the hypothalamic-pituitary-adrenal (HPA) axis, and likewise potentially the realisation of general inflammation due to stress through the same pathway[23]. This interaction provides a route by which stress reduction and better coping and resilience in the form of higher mastery might lead to reduced depression risk. It is possible that persons of greater mastery are more resilient and thus, less at risk of becoming depressed due to the effects of their disease. It is also possible that persons who are more resilient may represent a particular population for whom the inflammatory effects of disease do not realise as much mood disruption via the HPA axis. Both these interpretations would align with the prospective finding that pwMS of higher mastery were less likely to develop depression risk. Similarly, that no association was seen for loss of depression risk could suggest that those with the underlying preponderance to develop depression would not have a benefit from higher mastery because the disrupted lifestyle and inflammatory pathways conducive to depression are already present. Further exploration of both of these potential interpretations is warranted. If, however, the resilience interpretation is valid, this represents a point of intervention for pwMS to reduce their risk of depression. Through mindfulness or other mental health interventions, resilience and mastery could be increased and thence lead to a reduced risk of developing depression.

Our findings for other clinical outcomes assessed are less indicative of a true association, being only present crosssectionally for fatigue and disability but not prospectively, while for relapse number no robust associations were seen. Previous studies have reported similar findings of an association between fatigue and mastery and related concepts in pwMS. For example, in a cross-sectional study of 139 pwMS, higher environmental mastery, the ability to create an environment suitable to one's needs, assessed by a subdomain of the Ryff Happiness Scale [24], was inversely associated with fatigue severity [9]. For disability and mastery, less has been done. The aforementioned study by Schwartz and colleagues found no relationship between the Ryff Happiness Scale and disability as measured by EDSS [9]. Other prospective cohort studies of this relationship are yet needed to make definitive conclusions. 
That no associations were seen between mastery and relapse may be attributed to deficiencies in the measure. Participants were asked to report the number of doctor-diagnosed relapses they had experienced in the previous year. Such measures are unfortunately quite susceptible to recall bias and recall error, and a prospective measure of relapses would have been preferable. Thus, we are cautious in the interpretation of these results. There has been a no investigations of mastery and relapse risk in MS so we have no ability to compare with the literature on this topic. Other studies of the mastery-relapse relationship should be undertaken, ideally prospective in nature so as to better assess causality of associations, if any.

Strengths \& limitations

Our study has many strengths, comprising a large international cohort of pwMS, utilising a prospective study design, and including a diversity of demographic, clinical, lifestyle, and outcome measures. The sample size gives us significant statistical power. We acknowledge appreciable attrition from our baseline survey, with $34.0 \%$ retention. This is to some extent a function of the healthy participant bias attendant to many longitudinal epidemiological studies, but may reflect the online nature of recruitment that can impact on loss of contact due to changed email addresses as well as a lack of a more personal contact that come from clinic-based studies. Mastery was only measured at the 2.5-year timepoint onwards, therefore healthy participant bias is a potential confounder. Other limitations include the self-reported nature of the survey; however, for measures such as mastery and fatigue are necessarily subjective in nature, and validated tools were used where ever possible. Our prospective study design gives us the ability to assess causal directionality. The comprehensive data capture allows us to develop robust multivariable models that control for a great deal of potential confounding. Another potential limitation lies in not accounting for cognitive impairment as this might modulate the mastery-outcome relationship.

\section{Conclusion}

We have shown a strong positive association of higher mastery with less risk of depression, and a lower risk of developing depression in the future. Building mastery and resilience may thus represent a point of intervention to improve mental health in pwMS.

\section{Declarations}

\section{Funding}

This work was supported by anonymous philanthropic funders and Mr Wal Pisciotta. Open access publication was supported by the Overcoming Multiple Sclerosis Charity.

\section{Conflicts of interest}

GJ is the author of Overcoming Multiple Sclerosis and Recovering from Multiple Sclerosis, and the founder of the Overcoming MS Foundation. GJ and SN were facilitators of OMS educational workshops for people with MS.

\section{Acknowledgements}

We wish to acknowledge the generous philanthropic funders of the Neuroepidemiology Unit and the Overcoming Multiple Sclerosis Charity (open access publication). The funders and supporters had no role in any aspect of the research. We also wish to acknowledge the continued generosity of the HOLISM participants.

Availability of data 
Persons interested in acquiring the data underlying these analyses may contact Dr Sandra Neate or Dr Nupur Nag in regards and an anonymised data cut may be provided.

\section{Author contributions based on CRediT:}

Conceptualisation: GAJ, SN, NN, SSY; Methodology: SSY; Formal analysis: SSY, AH;

Investigation: GAJ, SN, NN, SSY; Resources: SN, NN; Data curation: SSY;

Writing - original: SSY, AH; Writing - revision SN, SSY, NN, AH; Manuscript approval - All authors; Supervision: SSY, NN;

Project administration: SN, NN; Funding acquisition: GAJ

\section{Ethics approval}

Ethics approval was given by The University of Melbourne's Health Sciences and Human Ethics Sub-Committee (HESC 1545102).

Consenting process

Participants were first presented with a patient information sheet, at the end of which they indicated if they consented to participate. If they selected yes, they proceeded to the survey. If they selected no, the survey closed.

\section{References}

1. Pearlin, L. I., \& Schooler, C. (1978). The Structure of Coping. Journal of Health and Social Behavior, 19(1), 2.

2. Bandura, A. (1977). Self-efficacy: toward a unifying theory of behavioral change. Psychol Rev, 84(2), 191-215.

3. Mikula, P., Nagyova, I., Vitkova, M., \& Szilasiova, J. (2018). Management of multiple sclerosis: the role of coping selfefficacy and self-esteem. Psychol Health Med, 23(8), 964-969.

4. Riazi, A., Thompson, A., \& Hobart, J. (2004). Self-efficacy predicts self-reported health status in multiple sclerosis. Mult Scler 10(1), 61-66.

5. Krokavcova, M., Nagyova, I., van Dijk, J. P., Rosenberger, J., Gavelova, M., Middel, B., Gdovinova, Z., \& Groothoff, J. W. (2008). Mastery, functional disability and perceived health status in patients with multiple sclerosis. . Eur J Neurol. , 15(11), 1237-1244.

6. Wassem, R. (1992). Self-efficacy as a predictor of adjustment to multiple sclerosis. J Neurosci Nurs 1992; 24: pp. 224229. J Neurosci Nurs 24, 224-229.

7. Motl, R. W., McAuley, E., \& Snook, E. M. (2007). Physical activity and quality of life in multiple sclerosis: possible roles of social support, self-efficacy, and functional limitations. Rehabil Psychol, 52, 143.

8. O'Kearney, E. L., Brown, C. R., Jelinek, G. A., Neate, S. L., Taylor, K. T., Bevens, W., De Livera, A. M., Simpson, S., Jr., \& Weiland, T. J. (2020). Mastery is associated with greater physical and mental health-related quality of life in two international cohorts of people with multiple sclerosis. Mult Scler Relat Disord, 38, 101481.

9. Schwartz, C. E., Coulthard-Morris, L., \& Zeng, Q. (1996). Psychosocial correlates of fatigue in multiple sclerosis. Arch Phys Med Rehabil, 77(2), 165-170.

10. Hart, S., Fonareva, I., Merluzzi, N., \& Mohr, D. C. (2005). Treatment for depression and its relationship to improvement in quality of life and psychological well-being in multiple sclerosis patients. . Qual Life Res. , 14(3), 695-703.

11. Hadgkiss, E. J., Jelinek, G. A., Weiland, T. J., Pereira, N. G., Marck, C. H., \& Van Der Meer, D. M. (2013). Methodology of an International Study of People with Multiple Sclerosis Recruited through Web 2.0 Platforms: Demographics, Lifestyle, and 
Disease Characteristics. Neurology Research International, 2013, 1-12.

12. Weiland, T. J., De Livera, A. M., Brown, C. R., Jelinek, G. A., Aitken, Z., Simpson, S. L., Neate, S. L., Taylor, K. L., O'Kearney, E., Bevens, W., \& Marck, C. H. (2018). Health Outcomes and Lifestyle in a Sample of People With Multiple Sclerosis (HOLISM): Longitudinal and Validation Cohorts. Frontiers in Neurology, 9.

13. Howe, L. D., Hargreaves, J. R., Ploubidis, G. B., De Stavola, B. L., \& Huttly, S. R. (2011). Subjective measures of socioeconomic position and the wealth index: a comparative analysis. Health Policy Plan, 26(3), 223-232.

14. Craig, C. L., Marshall, A. J., Sjöström, M., Bauman, A. E., Booth, M. L., Ainsworth, B. E., Pratt, M., Ekelund, U., Yngve, A., Sallis, J. F., \& Pekka, O. J. A. (2003). International Physical Activity Questionnaire: 12-country reliability and validity. Medicine \& Science in Sports \& Exercise, 35(8), 1381-1395.

15. Sangha, O., Stucki, G., Liang, M. H., Fossel, A. H., \& Katz, J. N. (2003). The Self-Administered Comorbidity Questionnaire: a new method to assess comorbidity for clinical and health services research. Arthritis Rheum, 49(2), 156-163.

16. Krupp, L. B., LaRocca, N. G., Muir-Nash, J., \& Steinberg, A. D. (1989). The fatigue severity scale. Application to patients with multiple sclerosis and systemic lupus erythematosus. Arch Neurol, 46(10), 1121-1123.

17. Hohol, M. J., Orav, E. J., \& Weiner, H. L. (1995). Disease steps in multiple sclerosis: a simple approach to evaluate disease progression. Neurology, 45(2), 251-255.

18. Kroenke, K., Spitzer, R. L., \& Williams, J. B. (2001). The PHQ-9: validity of a brief depression severity measure. J Gen Intern Med, 16(9), 606-613.

19. Simpson, S., Jr., Taylor, K. L., Jelinek, G. A., De Livera, A. M., Brown, C. R., O'Kearney, E., Neate, S. L., Bevens, W., \& Weiland, T. J. (2019). Associations of demographic and clinical factors with depression over 2.5-years in an international prospective cohort of people living with MS. Mult Scler Relat Disord, 30, 165-175.

20. Patten, S. B., Marrie, R. A., \& Carta, M. G. (2017). Depression in multiple sclerosis. International review of psychiatry, 29(5).

21. Tan-Kristanto, S., \& Kiropoulos, L. A. (2015). Resilience, self-efficacy, coping styles and depressive and anxiety symptoms in those newly diagnosed with multiple sclerosis. . Psychology, Health \& Medicine, 20, 635-645., 20, 635-645.

22. Berzins, S. A., Bulloch, A. G., Burton, J. M., Dobson, K. S., Fick, G. H., \& Patten, S. B. (2017). Determinants and incidence of depression in multiple sclerosis: A prospective cohort study. J Psychosom Res, 99, 169-176.

23. Feinstein, A., Magalhaes, S., Richard, J. F., Audet, B., \& Moore, C. (2014). The link between multiple sclerosis and depression. Nat Rev Neurol, 10(9), 507-517.

24. Ryff, C. D., \& Keyes, C. L. (1995). The structure of psychological well-being revisited. J Pers Soc Psychol, 69(4), $719-727$.

\section{Supplementary Files}

This is a list of supplementary files associated with this preprint. Click to download.

- STROBEchecklistcohort.docx

- SupplementalTables.docx 